www.jmscr.igmpublication.org

Impact Factor 5.244

Index Copernicus Value: 5.88

ISSN (e)-2347-176x ISSN (p) 2455-0450

crossref DOI:_http://dx.doi.org/10.18535/jmscr/v4i4.06

Journal Of Medical Science And Clinical Research

IGM Publication

An official Publication of IGM Publication

\title{
Cervical Dystonia and Psychiatric Manifestations in Hypoparathyroidism Associated with Extensive Intracerebral Calcifications
}

\author{
Authors \\ Prasanth.S.R ${ }^{1}$, Shaji.C. ${ }_{1,3,6}^{2}$, Parvathy.G ${ }^{3}$, Kabeer.K.A ${ }^{4}$, Ram Mohan ${ }^{5}$, Meenakumari.S \\ ${ }^{1,3,6}$ Senior Resident, ${ }^{2}$ Professor, ${ }^{4,5}$ Associate Professor, \\ Department of Neurology, Government TD Medical College, Alappuzha, Kerala, India \\ *Correspondence Author \\ Prasanth.S.R \\ Department of Neurology, Government TD Medical College, \\ Alappuzha, Kerala, India, PIN 688005 \\ Email: drprasanth2003@gmail.com
}

\begin{abstract}
Hypoparathyroidism can be associated with brain calcifications and varied neuropsychiatric manifestations. A 61 year old male presented with history of gradually progressive behavioural disturbances, initially diagnosed from outside hospitals as bipolar disorder and was on mood stabilisers since 40 years of age. Presently patient had episodic twisting movements of neck suggestive of cervical dystonia, relieved by sensory tricks, for last one year. History of occasional paraesthesias of extremities was present. No family history of similar illness.

Evaluation showed low serum calcium and parathormone levels. Renal function and ultrasound neck was normal with no history of prior thyroid surgery or irradiation. Neuroimaging revealed extensive intracerebral calcifications in bilateral basal ganglia, thalami, centrum semiovale and cerebellum. Patient was given oral calcium supplementation with anticholinergics leading to some relief of dystonia. Hypoparathyroidism can be difficult to diagnose due to frequent atypical presentations,often misdiagnosed as primary psychiatric illness as in our patient.Further, clinical presentation as isolated cervical dystonia is very rare.
\end{abstract}

Keywords- Hypoparathyroidism, cervical dystonia, neuropsychiatric manifestations, intracerebral calcifications.

\section{INTRODUCTION}

Hypoparathyroidism has varied neurological manifestations like numbness, parasthesias, seizures, behavioural changes, and extrapyramidal manifestations ${ }^{[1]}$. Atypical presentations lead to delay in diagnosis and often incorrect treatments. ${ }^{[2]}$ We report a case of hypoparathyroidism with secondary cervical dystonia and psychiatric manifestations in associated with extensive intracerebral calcifications.
This case highlights the importance of high index of suspicion and clinical differentiating features in atypical cases.

\section{CASE REPORT}

A 61 year old male presented with episodic involuntary painful abnormal posturing of neck for one year, precipitated by standing and walking, each episode lasting 1-3 minutes, relieved by sitting and reclining backwards in chair. 
Gradually progressive behavioural disturbances from the age of $40 \mathrm{yrs}$, initially began in the form of anxiety, excessive fear (fear of going out at night),abnormal sensations of extremities. There were frequent anger outbursts, even for trivial issues.Later he also developed paranoid delusions, that neighbours were trying to kill him, often slept with a knife under his pillow at night. He had episodes of severe depressive symptoms,crying spells, loss of sleep and appetite.

He was shown to several psychiatrists, was diagnosed as bipolar affective disorder and was treated with sodium valproate.

On examination patient was fully conscious well oriented, appeared anxious and fearful. Bilateral immature cataract were present. There were painful spasms of hand on inflating sphygmomanometer cuff above systolic blood pressure (i.e. Trousseau's sign was positive). Patient had persecutory delusions but there were no hallucinations. His memory and speech were normal. All the cranial nerves were intact. Motor power was normal in all four limbs and sensations were intact. Deep tendon reflexes were brisk with bilateral flexor plantar.

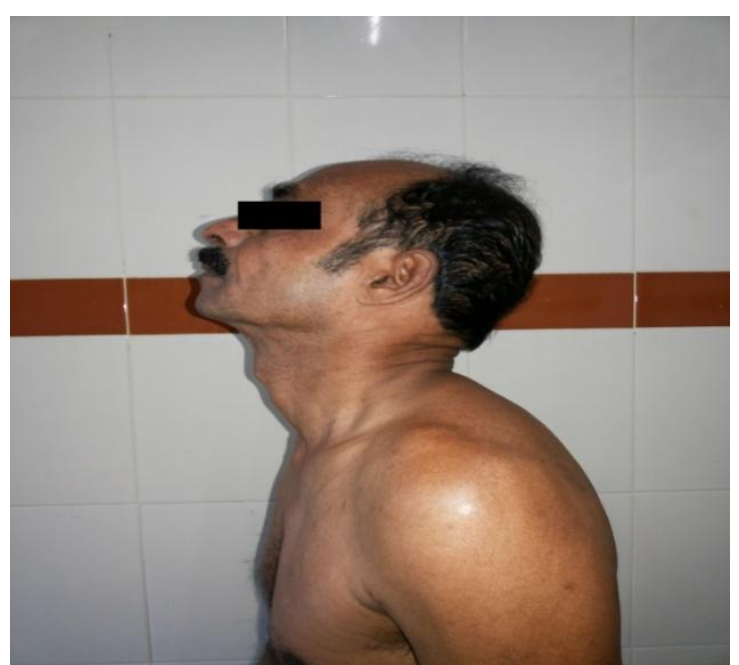

Figure 1: Patient having dystonic posturing of neck, predominantly laterocollis and retrocollis

There were involuntary movements in the form of twisting movements of neck with abnormal posture predominantly laterocollis and retrocollis, increased on standing and walking. [Figure 1]. It was reduced by sensory trick of sitting and reclining backwards in chair.
On investigation his complete blood count, blood sugar,renal and liver function tests were all within normal range. His serum albumin was $4.0 \mathrm{gm} / \mathrm{dl}$. Serum electrolytes were sodium $136 \mathrm{meq} / \mathrm{L}$, potassium $4.2 \mathrm{meq} / \mathrm{L}$, s. Calcium $7.9 \mathrm{mg} / \mathrm{dl}(\mathrm{N} 9$ 11)- repeatedly low, Phosphorus $5.4 \mathrm{mg} / \mathrm{dl}$ (N 2.54.6).24-hour urine calcium was $36 \mathrm{mg} / \mathrm{dl}(\mathrm{N}$ : 100300). His parathormone level was $3.51 \mathrm{pg} / \mathrm{ml}$ (normal range 15-68 pg/ml). Thyroid function tests were normal.

Ultrasound scan of neck and abdomen was normal. Slit lamp examination showed no KF ring.

CT scan of the brain revealed bilaterally symmetrical hyperdense lesions s/o calcification in basal ganglia, thalamus, white matter of cerebellar hemispheres and centrum semiovale. [Figure 2].MRI Brain showed bilateral symmetric T2 hypointensity with blooming in GRE in basal ganglia,thalami,cerebellar white matter, bilateral periventricular region and centrum semiovale [Figure 3].

On the basis of extrapyramidal features, bilateral cataract, positive Trousseau's sign, hypocalcemia, high phosphate levels and low PTH levels, patient was diagnosed as a case of hypoparathyroidism, possibly idiopathic. Calcitriol $0.5 \mu \mathrm{g} /$ day and calcium $1 \mathrm{gm} /$ day was started. Patient's calcium levels returned to normal range with this treatment. Patient had marked improvement in behavioural symptoms and slight improvement in dystonia. Trihexiphenidyl was also added to his regimen and kept under followup.

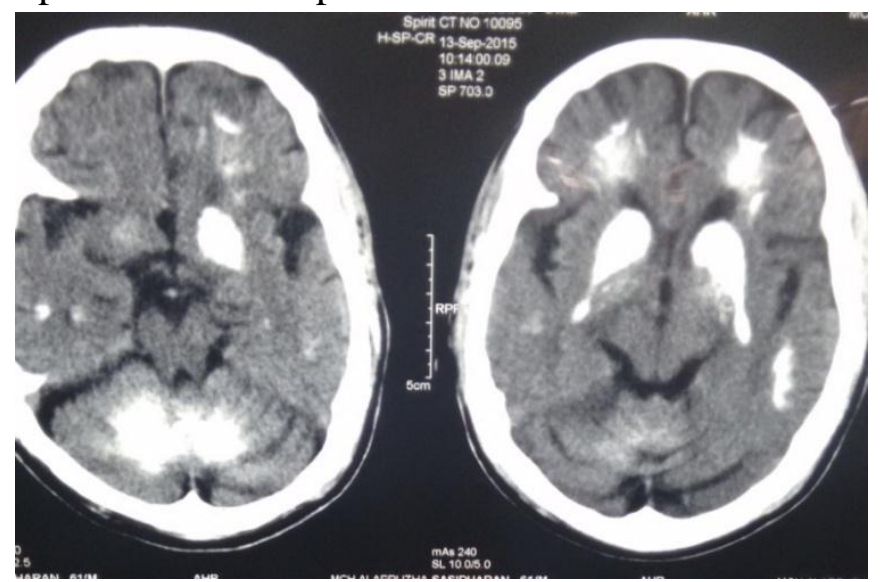

Figure 2: CT Brain showing bilateral symmetrical calcification in basal ganglia, thalamus, frontal subcortical white matter and cerebellar hemispheres. 


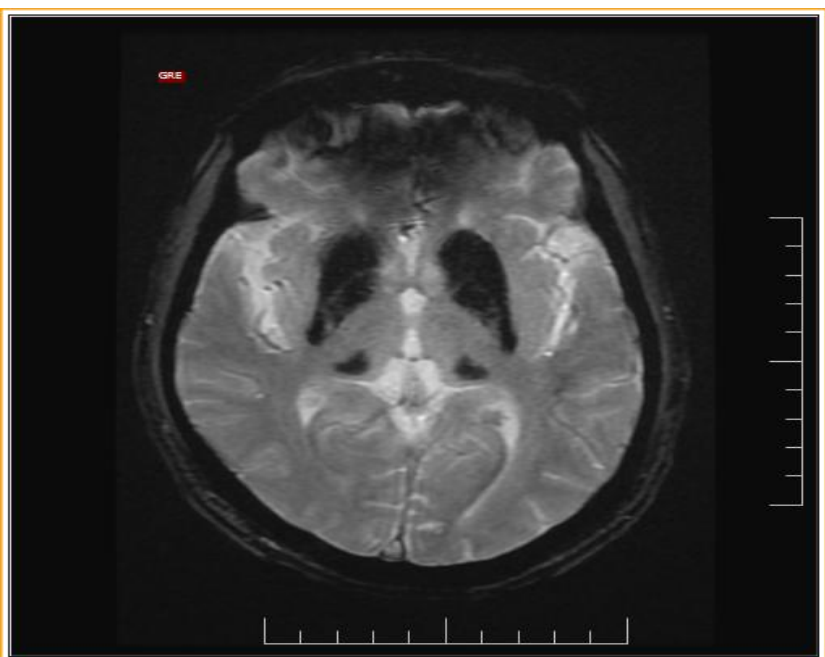

Figure 3: MRI Brain Gradient echo sequence showing blooming artefact suggestive of calcification in bilateral basal ganglia and thalamus.

\section{DISCUSSION}

Hypoparathyroidism usually presents with signs and symptoms of neuromuscular irritability, as manifestation of hypocalcemia ${ }^{[3]}$. However a myriad of atypical presentations can occur, which leads to diagnostic confusion. This is especially true with neuropsychiatric manifestations and extrapyramidal features ${ }^{[4]}$. Differentiation between a primary psychiatric disorder and hypoparathyroidism with neuropsychiatric symptoms may represent a challenge given that the classical manifestations of hypocalcemia may be absent in the setting of chronic hypoparathyroidism ${ }^{[5]}$.

These patients are often treated as primary psychiatric disorder and extrapyramidal features are attributed as drug induced. Especially in case of cervical dystonia ,this question arises, whether drug induced tardive type or due to hypoparathyroidism itself (termed as 'idiopathic' in literature) ${ }^{[6]}$.

Certain clinical points are helpful to distinguish idiopathic cervical dystonia from the tardive variety.In a study by Molho ES et al, torticollis, laterocollis, and trick maneuvers are predictive of idiopathic cervical dystonia. The presence of extracervical involvement and spasmodic head movements were individually found to be predictive of tardive cervical dystonia ${ }^{[7]}$. Clinical presentation of hypoparathyroidism as isolated cervical dystonia, as in our patient is very rare.
In a patient with neuropsychiatric symptoms and seconday cervical dystonia in the absence of neuroleptic drug exposure, a high index of clinical suspicion is needed for the correct diagnosis of hypoparathyroidism.

\section{REFERENCES}

1. Shoback D. Clinical practice. Hypoparathyroidism. N Engl J Med 2008; 359: 391-403.

2. Bhadada SK, Bhansali A, Upreti V, Subbiah S, Khandelwal N. Spectrum of neurological manifestations of idiopathic hypoparathyroidism and pseudohypoparathyroidism. Neurol India. 2011 Jul-Aug. 59(4):586-9.

3. Marx SJ. Hyperparathyroid and hypoparathyroid disorders. N Engl J Med. 2000 Dec 21. 343(25):1863-75.

4. El Otmani et al Striatopallidodentate calcinosis, hypoparathyroidism and neurological features: a case series study. Rev Neurol 2013 Jun-Jul;169(6-7): 495-501.

5. Idris MN, Sokrab TE. Unusual neuropsychiatric manifestations of hypoparathyroidism: report of two cases. East Afr Med J. 1998 Feb;75(2):127-8.

6. Mancini $F$ et al Secondary cervical dystonia in iatrogenic hypoparathyroidism associated with extensive brain calcifications. Funct Neurol. 2006, Jul-Sep;21(3):165-6.

7. Molho ES, Feustel PJ, Factor SA. Clinical comparison of tardive and idiopathic cervical dystonia. Mov Disord. 1998 May; 13(3): 486-9. 